\title{
Rigid Spine Syndrome: A Clinical Symptom Encompassing Various Aethiologies
}

\author{
Giovanni Antioco Putzu* \\ Department of Neurology and Clinical Neurophysiology, Italy
}

Submission: February 10, 2017; Published: February 17, 2017

*Corresponding author: Giovanni Antioco Putzu, Department of Neurology and Clinical Neurophysiology, Casa di Cura Polispecialistica

Sant’Elena, Viale Marconi 160, 09045 Quartu S Elena, Cagliari, Italy, Email: puzzugio@gmail.com

\begin{abstract}
The rigid spine syndrome (R.S.S.) is an unusual muscular disorder, which typical features include a reduction of the spine flexion and fibrous degeneration of axial muscles. This rare manifestation may be associated with miscellaneous conditions. We report two cases of R.S.S, one linked to desmine accumulation myopathy and another due to pseudohypoparathyroidism. Spinal muscle involvement was associated with muscular weakness of the limbs in the former; in contrast muscle strength was normal in the latter. Both had increased levels of creatine kinase (CK), respectively of $624 \mathrm{I} . \mathrm{U} / \mathrm{l}$ and $1648 \mathrm{I} . \mathrm{U} / \mathrm{l}$. To our knowledge the case of rigid spine syndrome associated with pseudohypoparathyroidism is unique, whereas the link of this syndrome with miscellaneous muscle disorders is much more usual. In addition, the pivotal role of calcium on CK increase is further clarify and confirmed by normalization of CKvalue after calcium therapy in the patient affected by pseudohypoparathyroidism. Although none had spinal deformities and thus, no indication for surgical correction, both are of clinical interest for the orthopaedics which may manage with patients presenting R.S.S.
\end{abstract}

Keywords: Rigid spine syndrome; Pseudohypoparathyroidism; Increased creatine kinase; Desmine myopathy

\section{Introduction}

The rigid spine syndrome (R.S.S.) is a unusual muscular disorder first defined in 1965 by Dubowitz [1,2] and later described by several others. It is a rare manifestation which typical features include a reduction of the spine flexion and fibrous degeneration of axial muscles. The main clinical features in this condition is a pronounced limitation of flexion of the cervical, thoracic and lumbar spine with the development of scoliosis eventually associated with contractures of others joints. Raised levels of C.K are frequently observed in R.S.S. associated with muscle disorders. Calcium is thought to have a pivotal role in controlling membrane permeability (ref.). An increase of CK, most probably due to leakage of intracellular enzyme has been observed in miscellaneous conditions presenting hypocalcemia as well (ref). Moreover a deficiency of phosphorylase a, as a cause of muscle weakness, has been well documented in this condition [1]. We further emphasize the different causes of RSS that may include myopathies or endocrine disturbances. Moreover, the unique case of pseudohypoparathyroidism associated with R.S.S. further clarify the different mechanisms which may lead to increased C.K level.

\section{Patients and Methods}

\section{Patient 1}

A 21 year-old boy, born from non-consanguineous healthy parents, presented with rigid spine syndrome, pelvic involvement with Gower's sign, scapular muscle strength deficit and a mild cognitive impairment. The patient, born at the 40th week of an uneventful pregnancy, was asymptomatic until the age of 15 although a mild spinal rigidity was complained early in infancy. Delivery occurs spontaneously and there was neither ischemic accident nor post-natal tetany. Developmental milestones were all normal. Walking occurred within a normal range Academic performances were poor. At admittance, the patient presented a waddling deambulation. Diagnostic procedures included physical examination, laboratory investigation, MRI of skeletal and cardiac muscles, Holter-E.C.G, electromyography, Welscher Adult Intelligence Scale (WAIS) test and muscle biopsy.

Muscle sample obtained from needle biopsy was snap-frozen in isopentane pre-cooled in liquid nitrogen. Cryostat sections 


\section{Current Trends in Clinical \& Medical Imaging}

(5 micron-thick) were processed for conventional histology using standard techniques. Immunohistochemistry was performed using a panel of antibodies directed against spectrin, dystrophin, alpha-sarcoglycane, beta-distro-glycane, and Major Histocompatibility Complex class I.

\section{Patient 2}

A 12 year-old boy presented with a rigid spine and an increased CK level (1648 UI/L) found by chance a few years before, during an assessment for a surgical intervention of tonsillectomy The patient never complained either of tetany or clinical manifestations clearly related to hypocalcemia. Familial history was negative for neuromuscular disorders. Personal history was also negative; the patient was born at the 40th week of an uneventful pregnancy. Delivery occurs spontaneously and there was neither ischemic accident nor post-natal titanic manifestations. Developmental milestones were all normal. Walking occurred within a normal age range. The patient lived a normal life, occasionally sporting (swimming). Academic performances were adequate. At age five, in order to undergo a surgical intervention, an increased CK level (600 UI/l) was observed.

At admittance, the patient complained a numbness of the lower limbs, but no frank tetany was mentioned. The patient also complained frequent episodes of diarrhoea. Diagnostic procedures included physical examination, laboratory investigation including serial C.K. evaluation, skeletal muscle CTscan, electromyography including ischemic test at the forearm, and needle muscle biopsy.

Muscle sample obtained from needle biopsy was snap-frozen in isopentane pre-cooled in liquid nitrogen. Cryostat sections (5 micron-thick) were processed for conventional histology using standard techniques. Immunohistochemistry was performed using a panel of antibodies directed against spectrine, dystrophine, alpha-sarcoglycane, beta-destro-glycane, and Major Histocompatibility Complex class I.

\section{Results}

\section{Patient 1}

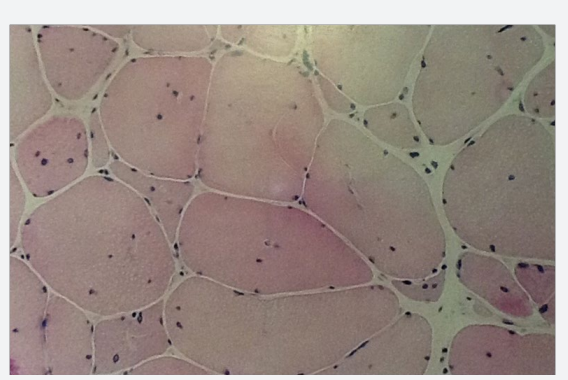

Figure 1: quadriceps muscle biopsy of patient 1 shows a great amount of internal nuclei, size variation of fibre size and atrophy of muscle fibres, Hematoxylin-Eosin stain, original magnification x200.
Ancillary results displayed a high CK value (624 I.U/l). Cardiac muscle MRI revealed a concentric hypertrophy of the left ventricle. There was no cardiac rhythm disturbances by HolterE.C.G. Muscle M.R.I was consistent with a fibrous degeneration of paravertebral muscles associated with psoas muscle involvement (Figure 1)

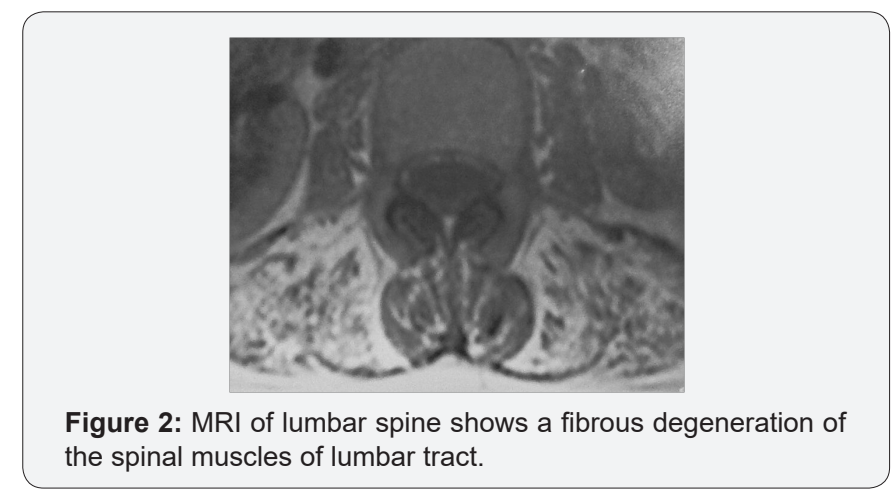

Muscle pathology of left quadriceps needle biopsy showed a severe fibrosis, an important fibre size variation with rare splitted and degenerated fibres. Internalized nuclei were present in almost 95\% of the fibres (Figure 2). Rimmed basophilic vacuoles were also noted, whereas acid phosphatase reaction was positive in most of the myofibres. Immunohistochemical reactivity to desmine was increased in vacuolated fibres. Electron microscopic examination revealed accumulation of a granulofilamentous material with desmine filament characteristics near the z-bands.

\section{Patient 2}

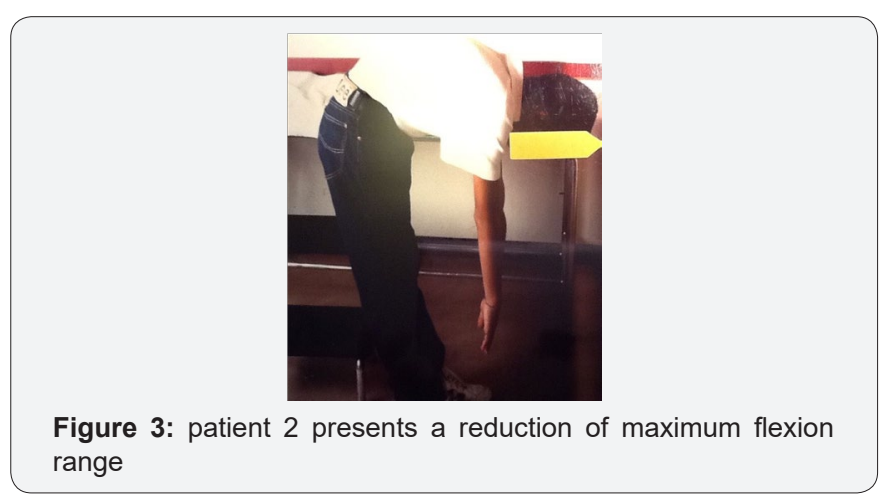

Clinical examination revealed a normal muscle bulk; medical research councyl (MRC) strentgh assessment was graded 5 in all muscles. Tendon reflexes were all normally elicited. Skeletal anomalies as pectus carenatum and mild scoliosis were observed. The patient had also an important reduction of the spine flexion, principally of the lumbar tract (Figure 3). Ischemic test at the forearm, performed using a cuff inflated above the diastolic pressure for 5 minutes was negative, but Chovsteck sign was positive. 
PTH serum level was particularly high (347 pg/ml), whereas calcium level was $5,2 \mathrm{mg} / \mathrm{dl}$.. No myogenic anomalies were observed in deltoid and quadriceps muscles by needle electromyography.

Serial CK dosages clearly showed that the enzyme dramatically decreased to half of the value three days after initiation of calcium therapy and was normal within one month of calcium therapy. The mechanism by which C.K. returned within normal range in a biphasic manner is discussed below.

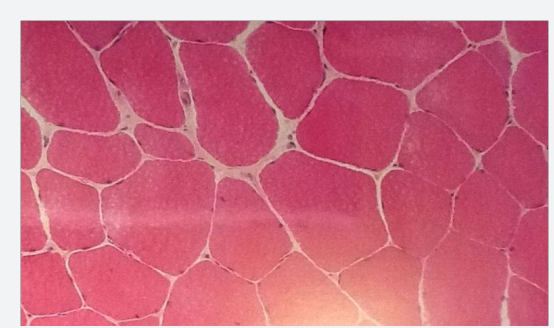

Figure 4: quadriceps muscle biopsy of patient 2: near-to-normal pattern. Only one atrophic fibre is observed, Hematoxylin-Eosin stain, original magnification $\times 100$.

CT scan of paravertebral muscles displayed an important atrophy associated with fibrous degeneration. Quadriceps muscle histology showed minimal anomalies characterised by a mild fibre size variation (Figure 4). Necrotic fibres were absent. Immunohistochemistry and electron microscopy were normal.

\section{Discussion}

To our knowledge, this is the first report of a R.S.S. associated with pseudohypoparathyroidism. Fibrous degeneration of para vertebral muscles, typical of R.S.S. has been observed by both ultrasound imaging and CT. Scan in patient 2. In contrast, R.S.S associated with different form of myopathy has been already reported $[3,4,5-10]$.

The dramatical improvement of CK level in patient 2 after initiating calcium supplementation and the minimal changes observed in the muscle biopsy, not concordant with the CK level, confirm the crucial effect of calcium on CK level as already demonstrated by Shane [9]. The biphasic improvement of CK value clearly demonstrates that the enzyme is linked to two different pools; a muscular one that rapidly returned to a normal value when calcium was supplemented and an osseous one requiring more time for normalisation since inversion of osteolytic process is not that immediate. In addition, we underline that osteolytic process, most probably because of the congenital condition, was quite limited in this patient, as indirectly proven by a mild increase of alcaline phosphatase level for age. Finally, we conclude that the major pool of CK is definitely represented by muscle $\mathrm{CK}$, whereas osseous $\mathrm{CK}$ is responsible for a slight, but more prolonged increase of the enzyme over time. Moreover, we emphasize that calcium metabolism has to be investigated when the cause of elevated CK level is obscure.

\section{Referen}

\section{ces}

1. Cape CA (1969) Phosphorylase a deficiency in pseudohypoparathyroidism. Neurology 19(2): 167-172.

2. Dubowitz V (1965) In Muscolar Dystrophy Research Group of Great Britain Third Symposium. Pitmann London, p. 57.

3. Flanigan KM, Kerr L, Bromberg MB, Leonard C, Tsuruda J et al, (2000) Congenital muscolar dystrofy, with rigid spine syndrome: a clinical, pathological, radiological, and genetic study. Ann Neurol 47(2): 152161.

4. Giannini S, Di Silvestre M, Ceccarelli F (1996) La Sindrome del Rachide Rigido. Progressi in patologia vertebrale, pp. 149-154.

5. Goebel HH, Halbig LE, Goldfarb L, Schober R, Albani M, et al, (2001) Reducing Body myopaty with cytoplasmic bodies and rigid spine syndrome: a mixed congenital myopaty. Neuropediatrics 32(4): 196205.

6. Palmucci L, Mongini T, Doriguzzi C, Schiffer D (1991) Familial autosomal recessive rigid spine with neurogenic facio-scapulo-peroneal muscle atrophy. J. Neurol Neurosurg Psychiatry 54(1): 42-45.

7. Sarnat HB (1995) Siblings with rigid spine syndrome and nemaline rod myopathy, a unique association. Neuromuscul Disord 5(4): 351-352.

8. Savini R, Cervellati S, Merlini L (1979) La sindrome del rachide rigido. Progressi in patologia vertebrale 2: 183-189.

9. Shane E, McClane KA, Olarte RM, Bilezikian JP (1980) Hypoparathyroidism and elevated muscle enzymes, Neurology 30(2): 192-195.

10. Topaloglu H, Gogus S, Yalaz K, Kucukali T, Serdaroglu (1994) Two

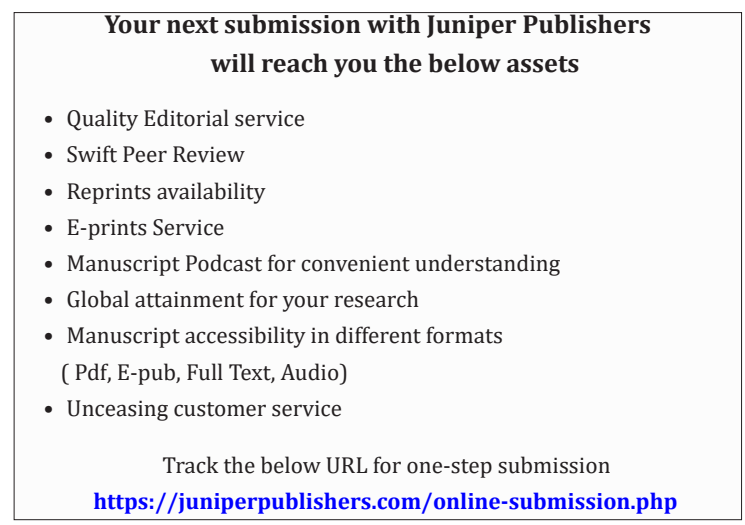

